\title{
An evaluation of the molecular mode of action of trans-resveratrol in the Porphyromonas gingivalis lipopolysaccharide challenged neuronal cell model
}

\author{
Bojlul Bahar $^{1}$ [D Sim K. Singhrao ${ }^{2}$
}

Received: 4 September 2020 / Accepted: 19 November 2020 / Published online: 8 December 2020

(c) The Author(s) 2020

\begin{abstract}
Porphyromonas gingivalis triggers a range of innate immune responses in the host that may contribute to the development of periodontitis and dementing diseases including Alzheimer's disease (AD). This study aimed to assess the mode of action of trans-resveratrol in modulating the $P$. gingivalis lipopolysaccharide (PgLPS) induced metabolic inflammation in a neuronal cell model. Confluent IMR-32 neuroblastoma cells were treated with trans-resveratrol from Polygonum cuspidatum in the presence or absence of PgLPS. The abundance of messenger ribo-nucleic acid (mRNA) transcripts of a panel of 92 genes was quantitatively assessed through targeted transcriptome profiling technique and the biochemical pathways affected were identified through Ingenuity Pathway Analysis. Gene expression analysis revealed that trans-resveratrol down-regulated the mRNA of multiple gene markers including growth factors, transcription factors, kinases, trans-membrane receptors, cytokines and enzymes that were otherwise activated by PgLPS treatment of IMR-32 neuroblastoma cells. Pathway analysis demonstrated that the cellular oxidative stress caused by the activation of phosphoinositide-3-kinase/Akt1 (PI3K/Akt1) pathway that leads to the production of reactive oxygen species (ROS), chronic inflammatory response induced by the activation of nuclear factor kappa-light-chain-enhancer of activated B cells (NF-kB) pathway and nutrient utilization pathways were favourably modulated by trans-resveratrol in the PgLPS challenged IMR-32 cells. This study demonstrates the potential of trans-resveratrol as a bioactive compound with multiple modes of intracellular action further supporting its therapeutic application in neuroinflammatory diseases.
\end{abstract}

Keywords Metabolic inflammation $\cdot$ Neuron $\cdot \mathrm{NF}-\mathrm{kB} \cdot$ Oxidative stress $\cdot$ Reactive oxygen species $\cdot$ LPS $\cdot$ Biochemical pathways

\section{Introduction}

Alzheimer's disease (AD) is a neurodegenerative disease characterized by the presence of insoluble amyloid-beta $(\mathrm{A} \beta)$ deposits called plaques and intraneuronal neurofibrillary tangles together with clinical signs of a deteriorating memory. AD has an inflammatory component that often

Bojlul Bahar

bbahar@uclan.ac.uk

1 International Institute of Nutritional Sciences and Food Safety Studies, School of Sport and Health Sciences, University of Central Lancashire, Preston PR1 2HE, Lancashire, UK

2 Brain and Behavior Centre, Faculty of Clinical and Biomedical Sciences, School of Dentistry, University of Central Lancashire, Preston, UK follows the development of the AD hallmark lesions. The microbial infections (bacteria/viruses) are implicated in the pathophysiology of $\mathrm{AD}$ and their relevance is the subject of current intensive research. We have reported the associations of Porphyromonas gingivalis lipopolysaccharide (PgLPS) in the brains of AD patients [1] and others have recently confirmed the presence of this bacterium in the brains of AD patients [2]. Further proof of concept studies in which, $P$. gingivalis was inoculated into the mouths of apolipoprotein E knock-out ( $\mathrm{APOE}^{--}$) mice demonstrated $P$. gingivalis bacterial DNA and LPS had entered the brains of $\mathrm{APOE}^{-/-}$mice [3], where the infection caused a significant increase in oxidative stress [4].

To understand the precise role of $P$. gingivalis in the development of hallmark lesions of AD, numerous studies performed oral infections with $P$. gingivalis or injected its lipopolysaccharide (LPS) in mice have supported the 
mechanism of inflammation that deteriorates further with aging $[5,6]$. Studies investigating either $P$. gingivalis or its outer membrane protein, PgLPS in vitro and in vivo $[2,3,5,7-9]$ suggest that this bacterium and its virulence factors play an important role in the pathophysiology of both periodontitis and AD. PgLPS is a potent immunogenic molecule that can alter immune homeostasis through altering the levels of the amyloid precursor protein, the parent protein of the $\mathrm{A} \beta$ plaque lesion in $\mathrm{AD}$. $\mathrm{AD}$ is a disease in which advancing age is a major risk factor and consequently, has the underlying inflammatory component deeply rooted in the innate immune system and metabolic inflammation. Hence, the precise role of the interplay of the innate immune and metabolic responses need to be better understood.

Resveratrol (3,4',5-trihydroxy-trans-stilbene) is a bioactive polyphenol naturally produced in plants such as grapes, berries, peanuts, tea and the root of Polygonum cuspidatum [10]. Trans-resveratrol has been reported to have numerous health benefits including anti-inflammatory, anti-diabetic, anti-oxidant and neuroprotective, and has been traditionally used in oriental medicine $[11,12]$. Growing evidence suggests that resveratrol has antimicrobial properties against a range of bacterial species, viruses and fungi [13]. Resveratrol inhibits the expression of virulence factor and interferes in biofilm formation where $P$. gingivalis is a keystone pathogen [14]. Resveratrol is a lipophilic polyphenol that can easily pass through the blood-brain barrier and hence is of particular interest for its bioactivity in neuronal health [15]. The neuroprotective role of trans-resveratrol against cellular oxidative damage has been previously reported [16]. In addition, several resveratrol derivatives have also been tested for their potential benefits in preventing neuronal cell death due to $A \beta$ deposition (an antimicrobial peptide in AD brains) taking place in the brain [17].

$P$. gingivalis can contribute to systemic and intracerebral pools of $\mathrm{A} \beta$ as well $[8,9]$. It is therefore plausible to suggest that $P$. gingivalis oral infection due to severe periodontitis has metabolic implications to an individual's peripheral and brain health. Neurons in the brain are vulnerable to oxidative stressors and a report by Kim et al. [18] suggested that resveratrol has a neuroprotective role by directly blocking the cellular oxidative stress caused by the release of reactive oxygen species (ROS). Resveratrol, despite being a highly bioactive molecule with a range of protective effects in the chronic inflammatory diseases, its therapeutic use in neuroinflammatory diseases particularly in the context of PgLPS induced oxidative stress and innate immune response are yet to be fully explored. This study aimed to assess the mode of action of trans-resveratrol in modulating the $P$. gingivalis LPS induced metabolic inflammation in the neuroblastoma IMR-32 in vitro cell model.

\section{Materials and methods}

\section{Cell culture}

The human neuroblastoma IMR-32 (ATCC) cell line was maintained in T25 flasks in Dulbecco's modified Eagle's medium (DMEM, Lonza UK) supplemented with $10 \%$ foetal bovine serum (FBS) Sigma-Aldrich, UK), $5 \mathrm{mM}$ Pen/strep, $5 \mathrm{mM}$ L glutamine, $5 \mathrm{mM}$ sodium pyruvate (all from Gibco UK). The cell culture media was replaced every other day and incubated at $37{ }^{\circ} \mathrm{C}$ in a humidified atmosphere of $5 \% \mathrm{CO}_{2}, 95 \%$ air until they reached about $90 \%$ confluence. The confluent cells were detached using $1 \mathrm{~mL}$ of $(1 \mathrm{x})$ trypsin (Gibco UK)/flask and pelleted by centrifugation. The cell density was determined using a Countess ${ }^{\circledR}$ cell counting chamber slides (ThermoFisher, Scientific, UK).

IMR-32 cells are relatively slow-growing cells with a population doubling time of approximately $48 \mathrm{~h}$. Fully confluent (>90\%) cells were used for PgLPS treatment in this study. For the treatment, the cells were seeded at $2 \times 10^{4}$ cells $/ \mathrm{mL}$ in a six-well plate. The cells reached a state of $>90 \%$ confluence within 6-7 days of seeding. On the day prior to treatment, the media was replaced with $1 \mathrm{~mL}$ serum and antibiotic-free media and cells were kept in the incubator for overnight. Then, cells were treated with $25 \mu \mathrm{M}$ of $98 \%$ trans-resveratrol (Kingherbs Ltd. Hunan, China) from P. cuspidatum in the presence/absence of $10 \mu \mathrm{g} / \mathrm{mL}$ commercial PgLPS from $P$. gingivalis (tlrlppglps, InvivoGen, France). The concentration of transresveratrol $(25 \mu \mathrm{M})$ was chosen based on the fact that resveratrol had no adverse effect on the cell viability and cytotoxicity up to $50 \mu \mathrm{M}[19,20]$. The PgLPS dose was selected based on the fact that a concentration of $10 \mu \mathrm{g} /$ $\mathrm{ml}$ PgLPS was reported to effectively induced intracellular oxidative and inflammatory cascades in murine macrophages [21]. The DMEM media without PgLPS served as a carrier control. Following $24 \mathrm{~h}$ incubation in the presence of PgLPS and resveratrol, the supernatants were removed and the cells were harvested in $1 \mathrm{ml}$ Trizol solution and stored at $-20{ }^{\circ} \mathrm{C}$ until used for RNA extraction.

\section{RNA extraction}

Total RNA was extracted from the cells using GenElute Total RNA Miniprep Kit (Sigma-Aldrich Corp.) according to the manufacturer's instructions. Total RNA was subjected to DNAse I (Sigma-Aldrich Corp.) treatment to eliminate genomic DNA contamination. Column purification of the RNA was performed using GenElute mammalian total RNA miniprep kit (Sigma-Aldrich Corp.). Total 
RNA was finally suspended in $50 \mu 10.1 \%$ diethylpyrocarbonate (DEPC) treated water and stored at $-80^{\circ} \mathrm{C}$. The quality and quantity of the total RNA were assessed in a NanoDrop-ND1000 Spectrophotometer (Thermo Fisher Scientific Inc. MA, USA). The cDNA synthesis was performed with $1 \mu \mathrm{g}$ of total RNA using the RevertAid $\mathrm{H}$ minus first-strand cDNA synthesis kit (Fermentas GmbH, St. Leon-Rot, Germany) following the manufacturer's protocol. The final volume of cDNA was adjusted to $150 \mu \mathrm{l}$ with nuclease-free water.

\section{Quantitative real-time PCR (qPCR)}

The expressions of a panel of 92 genes were evaluated using a qPCR array, performed in a QuantStudio 5 real-time PCR system (Applied Biosystems). This is a custom-designed 96-well array that included 92 target genes and four internal controls. The 92 target genes included representing the common markers of the inflammatory immune cascade. This panel of genes was previously reported to capture the intracellular signalling pathways associated with the chronic inflammation induced by LPS treatment [22]. Single internal control and 3 reference genes were also included on each plate. For this PCR array experiment, $25 \mu \mathrm{l}$ cDNA (after 1:5 dilution) from each replicate within a single treatment group was pooled. QPCR was performed on a $20 \mu \mathrm{l}$ reaction mixture per well containing $1 \mu \mathrm{l}$ pooled cDNA, $9 \mu \mathrm{l}$ water and $10 \mu \mathrm{l} \mathrm{SYBR}$ green master mix (Applied Biosystems). The thermal cycling conditions were $94{ }^{\circ} \mathrm{C}$ for $30 \mathrm{~s}$ followed by $60{ }^{\circ} \mathrm{C}$ for $1 \mathrm{~min}$, for 40 cycles. In this experiment, a CT value of 35 was considered as the cut-off limit. The relative quantities $\left(2^{-\Delta \mathrm{Ct}}\right)$ of the target genes were normalized using the geometric mean of the relative quantities of three internal control genes (beta-actin $(A C T B)$, hypoxanthine-guanine phosphoribosyl transferase 1 (HPRTI) and beta-2 microglobulin $(B 2 M)$. Briefly, average $\Delta \mathrm{Ct}$ was calculated as the difference of $\mathrm{Ct}$ values of any target gene minus the geometric average of the $\mathrm{Ct}$ value of the three reference genes. Then, fold change was calculated as $2^{(- \text {average }}$

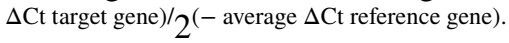

\section{Pathway Analysis}

The fold change values (cut off \pm 1.5 fold) were analyzed using Qiagen Ingenuity Pathway Analysis and the relevant canonical pathways were identified using the default setting in the software. The statistical probability ( $P$ value) of the observed number of genes affecting a particular biological function was calculated based on Fisher's Exact Test. This $P$ value indicated the statistical probability of the observed number of genes affected out of the total number of genes evaluated in the PCR array for the biological function. The correlation between the relationship direction and gene expression was determined by calculating the Z-score following the formula $\mathrm{Z}=\left(\mathrm{N}_{+}-\mathrm{N}_{-}\right) / \sqrt{ } \mathrm{N}$ where, $\mathrm{N}_{+}$represents the number of genes whose expression follows the same direction while N_represents the number of genes whose expression follows an opposite direction of the expression of a particular gene compared to that already available in the IPA knowledge database. $\mathrm{N}$ indicates the total number of genes affected. A high stringency Z-score between $\geq+2.0$ or $\leq-2.0$ were applied to identify the most relevant signalling pathways.

\section{Statistical analysis}

This experiment involved technical replicates on three independent occasions and an equal volume of cDNA was pooled from each of the three replicates representing the treatment group, the relative mRNA abundance values for each pool was considered as the mean for the treatment group.

\section{Results}

\section{Differential expression of genes by trans-resveratrol}

Of the 92 target genes evaluated, compared to the carrier control the PgLPS treatment resulted in the relative abundance of mRNA transcripts increased for 49 genes, decreased for 7 genes and no alteration in 36 genes (Fig. 1a). Among the key genes up-regulated by $>$ twofold (Table 1) were transcription factors (FOXO1, STAT1, STAT3, CREB1, EGR2, IRF1, FOS, RELA, NFKB1), kinases (AKT1, PIK3R1, GSK3B, PCK1, CSF1R, IRAK1, JAK2, MAPK3K1, $I K B K B, I N S R)$, receptors and associated protein (VCAMI, MYD88, CD40, TNFRSF1A, IGF1R, PTGER1, HRH3), enzymes (PTGS2, IRS1, TRAF2, ADA, FTO, HADH) and cytokines (TNFA, IL1B, CSF1, CSF2, IL6, IL8 and IL17A). The 7 genes down-regulated by PgLPS were GSTP1, SOD1, SOD2, TGFB1, RETN, ADIPOQ and LEPR.

Trans-resveratrol, in the presence of PgLPS challenge increased the mRNA transcript abundance of 19 genes, decreased of 44 genes with no alteration of 29 genes (Fig. 1b). Among the key 19 genes that were up-regulated by trans-resveratrol by a magnitude of $>$ twofold (Table 1 ) include ADIPOQ, IRS1, GSTP1, SOD1, SOD2, CSF1R, CD40, RETN, IGF1, EGR2, EGF, PCK1, IL17A, NOD2, CSF2, DPP4, MUC5AC, TGFB1 and LEPR. The 44 genes down-regulated by trans-resveratrol include transcription factors (CREB1, FOXO1, FOS, IRF1, JUN, NFKB1, RELA, STAT1, STAT3 and SREBEF1), kinases (AKT1, PIK3R1, GSK3B, IKBKB, INSR, IRAK1, IRAK2, JAK2 and MAP3KI), receptors (IGF1R, MYD88, IL6R, HRH3, TNFRSF1A, PTGERI and VCAMI), enzymes (ADA, FTO, HADH, IDE, 
Fig. 1 Relative abundance of mRNA transcripts of genes in the IMR-32 neuroblastoma cells subjected to Porphyromonus gingivalis lipopolysaccharide (PgLPS) treatment only (1a) or PgLPS and trans-resveratrol (1b)
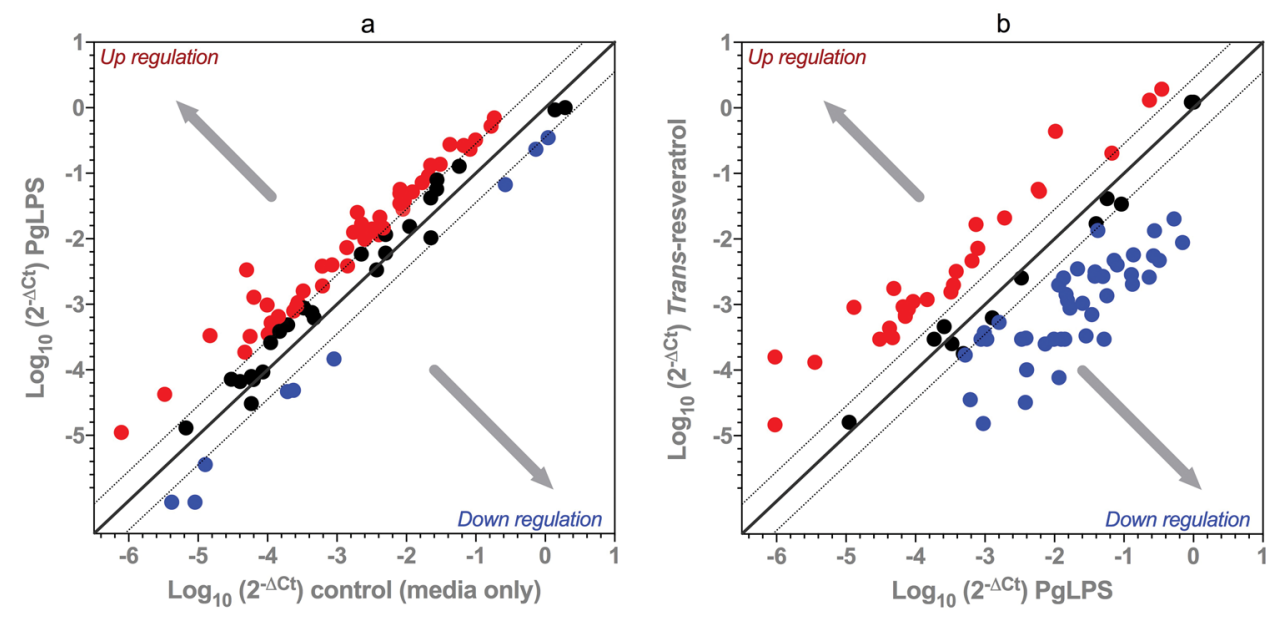

PLAT, PTGS2, and TRAF2) and cytokines (TNFA, IL6, ILIB and $C S F 1$ ).

\section{The biochemical pathways altered by trans-resveratrol in IMR-32 cells}

Based on the expression of genes differentially expressed by the trans-resveratrol treatment in PgLPS challenged IMR32 cells, the intracellular signalling pathways were identified (Table 2). Trans-resveratrol significantly $(\mathrm{P}<0.001$; $\mathrm{Z}$-score cut off $<-2.000$ ) inhibited pathways of cellular oxidative stress including the production of NO and ROS as indicated by the lower mRNA abundance of $A K T 1, F O S$, IKBKB, IRF1, JAK2, MAPK3K1, NFKB1, PIK3R1, RELA, STAT1, TNFA and TNFRSF1 genes and iNOS signaling as indicated by a decrease in the mRNA abundance of $F O S$, $I K B K B, I R A K 1, I R A K 2, I R F 1, J A K 2, N F K B 1, R E L A$ and $S T A T 1$ genes. Trans-resveratrol also inhibited the major inflammatory signalling pathways such as NF-kB signalling (18 genes), neuro-inflammatory signalling (22 genes), acute phase response (18 genes), IL-6 pathways (15 genes), IL-8 pathways (12 genes) and CD40 pathways (10 genes). For the nutrient metabolic pathways, trans-resveratrol activated PTEN pathways (9 genes), PPARa/RXRa pathways (10 genes) and PPAR signalling pathways (10 genes) while inhibited IGF-1 pathways (9 genes) and insulin receptor pathways ( 8 genes).

\section{Discussion}

Resveratrol is a bioactive polyphenol that has numerous health benefits including immuno-modulatory properties. The LPS of $P$. gingivalis (PgLPS), an anaerobic pathogen, implicated in periodontal disease induces innate immune responses that have far-reaching consequences in the immune and metabolic health of the host. In this study, the transcript abundance of a panel of key biomarkers altered by trans-resveratrol in the presence of PgLPS in IMR-32 neuroblastoma cells was evaluated. Gene expression analysis revealed that the markers of cellular oxidative stress, inflammatory pathways and glucose homeostasis as induced by the PgLPS treatment were altered by trans-resveratrol. This study demonstrates the potential of trans-resveratrol as a bioactive compound that has multiple modes of action including therapeutic potential in some neuroinflammatory diseases.

$P$. gingivalis and its LPS are predominantly found in the oral cavity, potentially contributing to dementing diseases of late-onset [1, 2, 7]. In Alzheimer's patients, the circulating plasma level of LPS was reported to be $61 \pm 42 \mathrm{pg} / \mathrm{ml}$ versus $21 \pm 6 \mathrm{pg} / \mathrm{ml}$ in the healthy volunteers [23]. In cell culture experiment, even a concentration of $10 \mathrm{ng} / \mathrm{ml}$ PgLPS caused only a mild response, however, a higher concentration of PgLPS (10 $\mu \mathrm{g} / \mathrm{ml})$ effectively induced intracellular oxidative and inflammatory cascades in murine macrophages [21]. Hence, PgLPS $10 \mu \mathrm{g} / \mathrm{ml}$ was used to treat the cells in this experiment. In this study, PgLPS treatment of IMR-32 cells resulted in a conspicuous up-regulation of key signalling molecules including transcription factors, kinases, membrane receptors, growth factors, enzymes and cytokines. Taken together, this data indicates the plausible activation of the oxidative and inflammatory cascades.

An LPS mediated activation of pro-inflammatory response is expected to occur through the involvement of pathogen recognition receptors (PRRs) molecules such as the toll-like receptors 4 (TLR-4) and 2 (TLR-2) and via MyD88 route leading to the activation of NF-kB [24]. This was evident from the increase mRNA abundance of MyD88, a number of genes of NF-kB pathway as well as major downstream inflammatory cytokines including TNFa, IL6 and IL1B indicating that PgLPS indeed induced the inflammatory signal cascade in IMR-32 cells. In periodontal inflammation, PgLPS was reportedly increased the levels of major inflammatory cytokines including TNFa, 
Table 1 Differential expression of genes caused by Porphyromonus gingivalis lipopolysaccharide (PgLPS) treatment only or PgLPS and transresveratrol in the IMR-32 neuroblastoma cells (fold change values $\geq 2.0$ and $\leq-2.0$ folds are shown)

\begin{tabular}{|c|c|c|c|c|}
\hline Genes & Location & Type of molecules & PgLPS & $\begin{array}{l}\text { PgLPS + trans- } \\
\text { resveratrol }\end{array}$ \\
\hline MYD88 innate immune signal transduction adaptor (MYD88) & Plasma membrane & Adapter protein & +4.87 & -2.96 \\
\hline Tumor necrosis factor alpha $(T N F A)$ & Extracellular space & Cytokine & +4.39 & -2.32 \\
\hline Interleukin 1 beta $(I L 1 B)$ & Extracellular space & Cytokine & +4.61 & -3.06 \\
\hline Colony stimulating factor $1(C S F 1)$ & Extracellular space & Cytokine & +5.34 & -29.33 \\
\hline Interleukin 6 (ILO) & Extracellular space & Cytokine & +67.17 & -11.30 \\
\hline Interleukin 8 (IL8) & Extracellular space & Cytokine & +22.51 & - \\
\hline Colony stimulating factor $2(C S F 2)$ & Extracellular space & Cytokine & +3.10 & +10.96 \\
\hline Interleukin 17A (IL17A) & Extracellular space & Cytokine & +2.60 & +9.82 \\
\hline Prostaglandin-endoperoxide synthase 2 (PTGS2) & Cytoplasm & Enzyme & +2.49 & -2.71 \\
\hline Insulin receptor substrate 1 (IRS1) & Cytoplasm & Enzyme & +5.77 & +4.78 \\
\hline Glutathione S-transferase pi 1 (GSTP1) & Cytoplasm & Enzyme & -3.13 & +5.51 \\
\hline TNF receptor associated factor $2(T R A F 2)$ & Cytoplasm & Enzyme & +4.16 & -12.25 \\
\hline Adenosine deaminase $(A D A)$ & Cytoplasm & Enzyme & +4.71 & -39.38 \\
\hline FTO alpha-ketogluterate dependent dioxygenase $(F T O)$ & Nucleus & Enzyme & +4.26 & -15.16 \\
\hline Superoxide dismutase 1 (SOD1) & Cytoplasm & Enzyme & -3.98 & +3.06 \\
\hline Superoxide dismutase 2 (SOD2) & Cytoplasm & Enzyme & -3.21 & +5.60 \\
\hline Hydroxyacetyle Co-A dehydrogenase $(H A D H)$ & Cytoplasm & Enzyme & +4.44 & -23.79 \\
\hline Prostaglandin E receptor 1 (PTGER1) & Plasma membrane & G-protein coupled receptor & +2.72 & -12.62 \\
\hline Histamine receptor $\mathrm{H} 3(H R H 3)$ & Plasma membrane & G-protein coupled receptor & +6.25 & -119.21 \\
\hline Epidermal growth factor $(E G F)$ & Extracellular space & Growth factor & - & +9.31 \\
\hline Insulin like growth factor $1(I G F 1)$ & Extracellular space & Growth factor & - & +8.78 \\
\hline Transforming growth factor beta $1(T G F B 1)$ & Extracellular space & Growth factor & -4.84 & +36.00 \\
\hline Vascular endothelial growth factor A (VEGFA) & Extracellular space & Growth factor & - & -13.35 \\
\hline Phosphoenol pyruvate carboxylase $1(P C K 1)$ & Cytoplasm & Kinase & +2.38 & +9.67 \\
\hline Colony stimulating factor 1 receptor $(C S F 1 R)$ & Plasma membrane & Kinase & +3.44 & +5.70 \\
\hline Interleukin 1 receptor associated kinase 1 (IRAK1) & Plasma membrane & Kinase & +3.26 & -68.45 \\
\hline Glycogen synthase kinase 3 beta $(G S K 3 B)$ & Nucleus & Kinase & +4.40 & -2.70 \\
\hline Janus kinase $2(J A K 2)$ & Cytoplasm & Kinase & +3.59 & -61.71 \\
\hline Phoshpoinositide-3 kinase regulatory subunit $1(P I K 3 R 1)$ & Cytoplasm & Kinase & +6.08 & -5.28 \\
\hline Mitogen activated protein kinase kinase kinase $1(M A P 3 K 1)$ & Cytoplasm & Kinase & +5.16 & -6.10 \\
\hline $\begin{array}{l}\text { Inhibitor of nuclear factor kappa B kinase subunit beta } \\
(I K B K B)\end{array}$ & Cytoplasm & Kinase & +3.14 & -10.25 \\
\hline Interleukin 1 receptor associated kinase 2 (IRAK2) & Plasma membrane & Kinase & - & -17.32 \\
\hline AKT serine/threonine kinase $1(A K T 1)$ & Cytoplasm & Kinase & +3.78 & -78.92 \\
\hline Insulin receptor $(I N S R)$ & Plasma membrane & Kinase & +4.38 & -47.27 \\
\hline Nuclear receptor subfamily 4 group A member 2(NR4A2) & Nucleus & Ligan-dependent nuclear receptor & +2.63 & -2.96 \\
\hline Dipeptidyl peptidase 4 (DPP4) & Plasma membrane & Peptidase & - & +12.00 \\
\hline Mucin 5AC, oligomeric mucus/gel-forming ( $M U C 5 A C)$ & Cytoplasm & Peptidase & - & +22.46 \\
\hline Plasminogen activator, tissue type (PLAT) & Extracellular space & Peptidase & +2.87 & -5.81 \\
\hline Insulin degrading enzyme (IDE) & Extracellular space & Peptidase & +7.03 & -41.87 \\
\hline Glucose-6 phosphatase catalytic subunit $(G 6 P C)$ & Cytoplasm & Phosphatase & +20.16 & -2.03 \\
\hline Early growth response 2 (EGR2) & Nucleus & Transcription regulator & +3.31 & +9.20 \\
\hline Interferon regulatory factor $1(I R F 1)$ & Nucleus & Transcription regulator & +2.32 & -151.71 \\
\hline Signal transducer and activator of transcription 1 (STAT1) & Nucleus & Transcription regulator & +4.08 & -14.09 \\
\hline cAMP responsive element binding protein $1(C R E B 1)$ & Nucleus & Transcription regulator & +6.13 & -18.41 \\
\hline Fos proto-oncogene, $\mathrm{AP}-1$ transcription factor subunit $(F O S)$ & Nucleus & Transcription regulator & +12.90 & -24.27 \\
\hline Forkhead box O1 (FOXO1) & Nucleus & Transcription regulator & +7.22 & -42.19 \\
\hline RELA proto-oncogene, NF-kB subunit $(R E L A)$ & Nucleus & Transcription regulator & +2.22 & -44.64 \\
\hline
\end{tabular}


Table 1 (continued)

\begin{tabular}{|c|c|c|c|c|}
\hline Genes & Location & Type of molecules & PgLPS & $\begin{array}{l}\text { PgLPS + trans- } \\
\text { resveratrol }\end{array}$ \\
\hline Nuclear factor kappa B subunit 1 (NFKB1) & Nucleus & Transcription regulator & +4.27 & -48.93 \\
\hline Signal transducer and activator of transcription 3 (STAT3) & Nucleus & Transcription regulator & +2.81 & -88.34 \\
\hline $\begin{array}{l}\text { Sterol regulatory element binding transcription factor } 1 \\
\quad(S R E B E F 1)\end{array}$ & Nucleus & Transcription regulator & +4.23 & -174.32 \\
\hline Jun proto-oncogene, AP1 transcription factor subunit $(J U N)$ & Nucleus & Transcription regulator & - & -3.12 \\
\hline Interleukin 6 receptor $(I L 6 R)$ & Plasma membrane & Trans-membrane receptor & +9.91 & -2.62 \\
\hline Leptin receptor $(L E P R)$ & Plasma membrane & Trans-membrane receptor & -2.17 & +42.48 \\
\hline CD40 molecule $(C D 40)$ & Plasma membrane & Trans-membrane receptor & +4.53 & +7.12 \\
\hline Vascular cell adhesion molecule 1 (VCAM1) & Plasma membrane & Trans-membrane receptor & +3.89 & -3.57 \\
\hline TNF receptor superfamily member $1 \mathrm{~A}(T N F R S F 1 A)$ & Plasma membrane & Trans-membrane receptor & +2.88 & -19.77 \\
\hline Insulin like growth factor 1 receptor $(I G F 1 R)$ & Plasma membrane & Trans-membrane receptor & +5.89 & -64.43 \\
\hline Uncoupling protein $2(U C P 2)$ & Cytoplasm & Transporter & +6.51 & -20.67 \\
\hline TNF receptor associated factor 5 (TRAF5) & Cytoplasm & Transporter & +3.91 & -33.05 \\
\hline Low density lipoprotein receptor $(L D L R)$ & Plasma membrane & Transporter & +3.19 & -85.47 \\
\hline $\begin{array}{l}\text { Nucleotide binding oligomerization domain containing } 2 \\
\text { (NOD2) }\end{array}$ & Cytoplasm & - & - & +10.84 \\
\hline Resistin $(R E T N)$ & Extracellular space & - & -6.15 & +8.10 \\
\hline $\begin{array}{l}\text { Adiponectin, } \mathrm{C} 1 \mathrm{Q} \text { and collagen domain containing (ADI- } \\
P O Q)\end{array}$ & Extracellular space & - & -4.14 & +6.66 \\
\hline GRB2 associated binding protein $1(G A B 1)$ & Cytoplasm & - & +7.40 & -18.91 \\
\hline
\end{tabular}

Table 2 Canonical pathways and the gene affected by the trans-resveratrol in presence of Porphyromonus gingivalis lipopolysaccharide (PgLPS) treatment in the IMR-32 neuroblastoma cells

\begin{tabular}{|c|c|c|c|c|}
\hline Pathways & Effect & Genes affected within the pathway & Z-score & P-value \\
\hline \multicolumn{5}{|l|}{ Oxidative stress } \\
\hline Production of NO and ROS & Inhibition & $\begin{array}{l}\text { AKT1, FOS, IKBKB, IRF1, JAK2, MAP3K1, NFKB1, PIK3R1, RELA, STAT1, } \\
\text { TNF, TNFRSF1 }\end{array}$ & -2.309 & $1.51 \mathrm{E}-13$ \\
\hline iNOS signaling & Inhibition & FOS, IKBKB, IRAK1, IRAK2, IRF1, JAK2, NFKB1, RELA, STAT1 & -2.330 & $6.10 \mathrm{E}-17$ \\
\hline \multicolumn{5}{|l|}{ Inflammation } \\
\hline NF-kB signaling & Inhibition & $\begin{array}{c}A K T 1, C D 40, E G F, G S K 3 B, I G F 1 R, I K B K B, I L 1 B, I N S R, I R A K 1, M A P 3 K 1, \\
\text { MYD88, NFKB1, PIK3R1, RELA, TNFA, TNFRSF1A, TRAF2, TRAF5 }\end{array}$ & -2.089 & $2.02 \mathrm{E}-27$ \\
\hline Neuroinflammation signaling & Inhibition & $\begin{array}{l}\text { AKT1, CD40, CREB1, CSF1R, FOS, GSK3B, IDE, IKBKB, IL6, IL1B, IL6R, } \\
\text { IRAK1, IRAK2, JAK2, MYD88, NFKB1, PIK3R1, PTGS2, RELA, SOD2, } \\
\text { STAT1, TGFB1 }\end{array}$ & -2.000 & $2.70 \mathrm{E}-32$ \\
\hline Acute phase response & Inhibition & $\begin{array}{l}\text { AKT1, FOS, IKBKB, IL6, IL1B, IL6R, IRAK1, JAK2, MAP3K1, MYD88, NFKB1 } \\
\text { PIK3R1, RELA, SOD2, STAT3, TNF, TNFRSF1A, TRAF2 }\end{array}$ & -2.186 & $9.55 \mathrm{E}-24$ \\
\hline IL-6 signaling & Inhibition & $\begin{array}{l}\text { AKT1, FOS, IKBKB, IL6, IL1B, IL6R, JAK2, NFKB1, PIK3R1, RELA, STAT3, } \\
\text { TNF, TNFRSF1A, TRAF2, VEGFA }\end{array}$ & -2.324 & $3.89 \mathrm{E}-21$ \\
\hline IL-8 signaling & Inhibition & $\begin{array}{l}\text { AKT1, EGF, FOS, IKBKB, IRAK1, IRAK2, NFKB1, PIK3R1, PTGS2, RELA, } \\
\text { VCAM1, VEGFA }\end{array}$ & -2.111 & $2.75 \mathrm{E}-13$ \\
\hline CD40 signaling & Inhibition & CD4O, FOS, IKBKB, NFKB1, PIK3R1, PTGS2, RELA, STAT3, TRAF2, TRAF5 & -2.333 & $1.61 \mathrm{E}-15$ \\
\hline \multicolumn{5}{|l|}{ Metabolism } \\
\hline PTEN signaling & Activation & AKT1, FOXO1, GSK3B, IGF1R, IKBKB, INSR, NFKB1, PIK3R1, RELA & 2.121 & $6.43 \mathrm{E}-11$ \\
\hline PPARa/RXRa activation & Activation & $A D I P O Q, I K B K B, I L 6, I L 1 B, I N S R, I R S 1, J A K 2, N F K B 1, R E L A, T G F B 1$ & 2.111 & $4.61 \mathrm{E}-12$ \\
\hline PPAR signaling & Activation & FOS, IKBKB, IL1B, INSR, NFKB1, PTGS2, RELA, TNF, TNFRSF1A, TRAF2 & 2.001 & $4.26 \mathrm{E}-15$ \\
\hline IGF-1 signaling & Inhibition & AKT1, FOS, FOXO1, IGF1, IGF1R, IRS1, JAK2, PIK3R1, STAT3 & -2.121 & $2.63 \mathrm{E}-13$ \\
\hline Insulin receptor signaling & Inhibition & AKT1, FOXO1, GAB1, GSK3B, INSR, IRS1, JAK2, PIK3R1 & -2.017 & $1.97 \mathrm{E}-10$ \\
\hline
\end{tabular}


IL-6, IL-1B and IL-17 [25, 26]. These pro-inflammatory cytokines can potentially contribute to the development of a systemic chronic inflammatory condition and pathophysiology of other inflammatory diseases such as cardiovascular diseases and type- 2 diabetes [3,5]. Thus, an unhygienic oral environment with generalised periodontitis may negatively affect a number of human inflammatory, but chronic diseases because of the common signalling cascades being activated. The drive to combat chronic diseases, which lower the individual's quality of life, is to improve lifestyle habits [27, 28].

In this study, the anti-oxidative and anti-inflammatory effects of trans-resveratrol were evident from the downregulation of a number of key regulatory kinases, transmembrane receptors, enzymes and inflammatory cytokines all together indicating a potential therapeutic application of resveratrol in $P$. gingivalis mediated pro-inflammatory disease that may eventually lead to AD. Resveratrol (3,5,4'-trihydroxy-trans-stilbene), a polyphenol naturally present in many plant components and long known for its anti-oxidative, anti-inflammatory properties and its role in glucose metabolism, has been used in the oriental medicine for centuries [29]. We identified that the anti-oxidative activity of trans-resveratrol is mediated through inhibition of two vital oxidative stress pathways (production of nitric oxide (NOS) and reactive oxygen species (ROS) and iNOS signalling pathways. ROS are oxygen-containing molecules produced as a result of cellular metabolism and are required for facilitating the transfer of electrons in the redox reactions as well as serving as a second messenger [30]. Uncontrolled oxidative stress is associated with metabolic inflammation as well as neurodegenerative diseases such as $\mathrm{AD}[31,32]$. In $\mathrm{AD}, \mathrm{ROS}$ interacting with $\mathrm{A} \beta$ leads to the production and accumulation of toxic compounds and plaque formation ultimately leading to synaptic loss and a compromised cognitive function in $\mathrm{AD}$ pathogenesis [30,32]. Another mechanism of ROS in AD pathogenesis involves its contribution towards the progressive loss of neuronal autophagy, a vital mechanism through which cell degrades the cytoplasmic proteins and organelles [30,33].

Underlying bioactivity of trans-resveratrol could be mediated via the inhibition of phosphor-inositol 3 kinase (PI3K)/ AKT1 pathway because the mRNA abundance of PI3KR1, a regulatory PI3K, along with AKT1, a serine/threonine kinase were inhibited by trans-resveratrol in the presence of PgLPS. The PI3K/AKT1 pathway plays an important role in the cellular oxidation process and contributes to the pathophysiology of neurodegenerative diseases [34]. While the PI3K/AKT pathway is indispensable for maintaining the homeostatic level of cell growth, survival and differentiation and metabolism of nutrients [34], during the cellular oxidative stress PI3K together with AKT1, trigger the production of ROS [35, 36]. AKT1 is involved in the phosphorylation of a number of signalling molecules such as glycogen synthase kinases 3 beta (GSK-3B), forkhead box class O1 (FOXO1) and NF-kB [34]. Along with PI3KR1 and AKT1, the mRNA abundance of these markers was also down-regulated by trans-resveratrol in this experiment. An LPS-mediated inflammatory response mediated through its receptor molecule TLR4 is also associated with the activation of AKT1 [37]. However, in this experiment, no change in the TLR4 mRNA was evident while cells were treated with trans-resveratrol in the presence of PgLPS. A potentially underlying cause could be that because the cells were harvested $24 \mathrm{~h}$ post-treatment, a time scale regarded sufficient to dampen the TLR 4 expression caused by a higher level of AKT1 expression over time [38].

The bioactivities of trans-resveratrol are known to affect the oxidative stress pathways, systemic inflammatory signalling and glucose metabolic pathways [39, 40]. Such broad-spectrum bioactivities of trans-resveratrol was exhibited by affecting the GSK-3B, an important regulatory molecule involved in the phosphorylation of a number of proteins and transcription factors involved in these three interconnected pathways [34, 40]. In addition to the PgLPS mediated inflammatory process, GSK-3B also contributes to excessive phosphorylation of the microtubule binding protein tau which eventually caused their collapse leading to neurofibrillary tangle formation, which is the second diagnostic hallmark of AD neuropathology [30, 41]. Another mechanism through which GSK-3B promotes the inflammatory process is through phosphorylation of STAT3, an important transcription factor required for the induction of pro-inflammatory cytokines that translocate into the nucleus following phosphorylation and activation [41]. Transcription factor CREB1 involved in the TLR mediated inflammation serves as a substrate for GSK-3B [40]. The importance of GSK-3B molecule in LPS mediated inflammatory response was demonstrated by the fact that inhibition of GSK-3B effectively saved mice from the deleterious effect of a lethal doze of LPS [42]. In this experiment together with GSK3B, the mRNA abundance of STAT3 and CREB1 were also down-regulated indicating the therapeutic application of trans-resveratrol in PgLPS mediated deleterious pro-inflammatory response.

GSK-3B also plays a major role in the nutrient metabolism through directly interacting with insulin and mTOR molecules and directing the nutrient energy towards the anabolic path leading to cell growth [40]. Expression of GSK-3B is vital for homeostasis as the complete elimination of GSK-3B is fatal for cell survival as demonstrated in the knockdown embryonic model [42]. In contrast, a temporary inhibition was found to increase in glucose uptake as well as a higher expression of GLUT1 [43]. GSK-3B also functions as a negative regulator of mitochondrial energy production that is mediated through controlling the AMPK, a cellular energy-sensing molecule, and inhibition of pyruvate 
dehydrogenase activity [44]. In this experiment, while the mRNA levels of G6P, an entry point of glucose into the energy metabolism and mitochondrial uncoupling protein 2 (UCP2) were inhibited by trans-resveratrol; two other markers of glucose metabolism (IRS1 and PCK1) remained unaffected. This potentially complex mechanism of transresveratrol action in nutrient metabolism via affecting GSK3B warrants further investigation.

FOXO1 belongs to the FoxO, forkhead box class $\mathrm{O}$ (FoxO) cluster of transcription factors that phosphorylates and acetylates proteins at serine, threonine and lysine residues and is involved in the oxidative stress and insulin action $[34,45]$. Both insulin resistance and oxidative stress can enhance the transcriptional activity of FOXO1 that can again contribute to the development of hyperglycemia and the production of ROS [46]. FOXO1 can also activate c-Jun $\mathrm{N}$-terminal kinase and inhibit Wingless (Wnt) pathways and can contribute to $\beta$-amyloid plaque formation and phosphorylation of s protein potentially leading to neurodegeneration [46]. In response to the $P$ gingivalis infection that often results in a compromised barrier function of the mucosal/ gingival epithelium, the PgLPS is expected to increase the FOXO1 expression [45]. Then, a subsequent translocation of FOXO1 into the nucleus is expected to cause transcriptional activation of a number of inflammatory mediators bearing a FOXO1 promoter responsive element [45]. FOXO1 activates a number of downstream molecules involved in the wound healing process of the epithelial barrier [47]. In this experiment, in addition to a reduction in the mRNA abundance of FOXO1, trans-resveratrol also down-regulated the abundance of few interesting downstream molecules such as pro-inflammatory cytokines (IL1B, TNFA), wound healing factor (VEGF) and adhesion molecule (VCAM1). TGFB is another FOXO1 target molecule that plays a vital role in the wound healing process [45]. Trans-resveratrol increased the mRNA abundance of TGFB suggesting its beneficial role in the PgLPS induced inflammatory damage.

Another interesting molecule with multiple protective functions in cell survival and metabolism is the phosphatase and tensin homolog (PTEN), a tumor suppressor that antagonizes phosphatidylinositol 3-kinase type I (PI3K) and thus affecting PI3K/AKT pathways [48]. PTEN is a tumour suppressor molecule that catalyses converting of PIP3 to PIP2 and reverses the PI3K/AKT activities. PTEN can increase energy expenditure in brown adipose tissue and enhance longevity at organism level indicating that an increase in PTEN activity could be beneficial [49]. One of the bioactivity of trans-resveratrol includes up-regulation of the PTEN pathway [48] that is associated with concomitant inhibition of PI3K/AKT functions. An outcome of this response is an up-regulation of genes involved in triggering the antioxidant pathways such as catalases and superoxide dismutase enzymes [48]. Our pathway analysis identified the activation of PTEN pathways as a potential mechanism of underlying anti-oxidant bioactivities of trans-resveratrol which is also conspicuous in the down-regulation of the mRNA abundance of major genes of PTEN pathway including AKT1, FOXO1, GSK3B, PIK3R1 and NF-kB associated markers. Besides, a potential triggering of genes involved in the antioxidant mechanism such as SOD1, SOD2 and GSTP1 [50] was also evident in this study further demonstrating multicomponent mechanisms underlying the trans-resveratrol mediated bioactivity in IMR-32 cells.

In conclusion, this study of targeted transcriptome profiling identified a number of biochemical signalling pathways affected in the IMR-32 neuroblastoma cells when treated with PgLPS. The gene expression analysis revealed that the trans-resveratrol has multiple highly effective bioactivities that work through modulating the interconnected mechanisms of cellular oxidative stress, inflammatory response and nutrient metabolism. Hence, the therapeutic application of trans-resveratrol in P. gingivalis mediated disease pathophysiology may be explored. Based on the altered expression of multiple markers of cellular oxidative stress, inflammatory pathways and nutrient metabolism, the PgLPS treated IMR-32 cells may be a good in-vitro model of $P$. gingivalis induced innate immune cascade that could eventually lead to the development of AD.

Acknowledgement This research is supported by a PreViser award in 2018 from the Oral and Dental Research Trust.

Author contributions BB conceived and designed the study, analysed data and wrote the manuscript. SS contributed in designing the study, performed lab work and contributed writing the manuscript.

Funding This research was supported by a PreViser award in 2018 from the Oral and Dental Research Trust.

\section{Compliance with ethical standards}

Conflicts of interest The authors declare no conflict of interest/competing interests for this work.

Open Access This article is licensed under a Creative Commons Attribution 4.0 International License, which permits use, sharing, adaptation, distribution and reproduction in any medium or format, as long as you give appropriate credit to the original author(s) and the source, provide a link to the Creative Commons licence, and indicate if changes were made. The images or other third party material in this article are included in the article's Creative Commons licence, unless indicated otherwise in a credit line to the material. If material is not included in the article's Creative Commons licence and your intended use is not permitted by statutory regulation or exceeds the permitted use, you will need to obtain permission directly from the copyright holder. To view a copy of this licence, visit http://creativecommons.org/licenses/by/4.0/. 


\section{References}

1. Poole S, Singhrao SK, Kesavalu L, Curtis MA, Crean S (2013) Determining the presence of periodontopathic virulence factors in short-term post-mortem Alzheimer's disease brain tissue. J Alzheimers Dis 36:665-677

2. Dominy SS, Lynch C, Ermini F, Benedyk M, Marczyk A, Konradi A, Nguyen M, Haditsch U, Raha D, Griffin C, Holsinger LJ, Arastu-Kapur S, Kaba S, Lee A, Ryder MI, Potempa B, Mydel P, Hellvard A, Adamowicz K, Hasturk H, Walker GD, Reynolds EC, Faull RLM, Curtis MA, Dragunow M, Potempa J (2019) Porphyromonas gingivalis in Alzheimer's disease brains: Evidence for disease causation and treatment with small-molecule inhibitors. Sci Adv 5(1):eaau3333. https://doi.org/10.1126/sciadv.aau3333

3. Poole S, Singhrao SK, Chukkapalli S, Rivera M, Velsko I, Kesavalu L, Crean S (2015) Active invasion of Porphyromonas gingivalis and infection-induced complement activation in ApoE/- mice brains. J Alzheimers Dis 43:67-80

4. Rokad R, Moseley R, Hardy SR, Chukkapalli S, Crean S, Kesavalu L, Singhrao SK (2017) Cerebral oxidative stress and microvasculature defects in TNF- $\alpha$ expressing transgenic and Porphyromonas gingivalis-infected $\mathrm{ApoE}^{-/-}$mice. J Alzheimers Dis 60:359-369

5. Wu Z, Ni J, Liu Y, Teeling JL, Takayama F, Collcutt A, Ibbett P, Nakanishi H (2017) Cathepsin B plays a critical role in inducing Alzheimer's disease-like phenotypes following chronic systemic exposure to lipopolysaccharide from Porphyromonas gingivalis in mice. Brain Behav Immun 65:350-361. https://doi.org/10.1016/j. bbi.2017.06.002

6. Zhang J, Yu C, Zhang X, Chen H, Dong J, Lu W, Song Z, Zhou W (2018) Porphyromonas gingivalis lipopolysaccharide induces cognitive dysfunction, mediated by neuronal inflammation via activation of the TLR4 signaling pathway in C57BL/6 mice. J Neuroinflam 15:37. https://doi.org/10.1186/s12974-017-1052-x

7. Ilievski V, Zuchowska PK, Green SJ, Toth PT, Ragozzino ME, Le K, Aljewari HW, O'Brien-Simpson NM, Reynolds EC, Watanabe K (2018) Chronic oral application of a periodontal pathogen results in brain inflammation, neurodegeneration and amyloid beta production in wild type mice. PLoS ONE 13(10):e0204941. https ://doi.org/10.1371/journal.pone.0204941

8. Nie R, Wu Z, Ni J, Zeng F, Yu W, Zhang Y, Kadowaki T, Kashiwazaki H, Teeling JL, Zhou Y (2019) Porphyromonas gingivalis infection induces amyloid- $\beta$ accumulation in monocytes/macrophages. J Alzheimers Dis 72:479-494

9. Zeng F, Liu Y, Huang W, Qing H, Kadowaki T, Kashiwazaki H, Ni J, Wu Z (2020) Receptor for advanced glycation end products upregulation in cerebral endothelial cells mediates cerebrovascularrelated amyloid $\beta$ accumulation after Porphyromonas gingivalis infection. J Neurochem. https://doi.org/10.1111/jnc.15096

10. Soleas GJ, Diamandis EP, Goldberg DM (1997) Wine as a biological fluid: history, production, and role in disease prevention. J Clin Lab Anal 11:287-313

11. Baur JA, Sinclair DA (2006) Therapeutic potential of resveratrol: The in vivo evidence. Nat Rev Drug Discov 5:493-506

12. Gandhi H, Rathore C, Dua K, Vihal S, Tambuwala MM, Negi P (2020) Efficacy of resveratrol encapsulated microsponges delivered by pectin based matrix tablets in rats with acetic acid-induced ulcerative colitis. Drug Dev Ind Pharm 46:365-375. https://doi. org/10.1016/j.ijpharm.2020.119531

13. Vestergaard $M$, Ingmer $H$ (2019) Antibacterial and antifungal properties of resveratrol. Int J Antimicrob Agents 53:716-723. https://doi.org/10.1016/j.ijantimicag.2019.02.015

14. Kugaji MS, Kumbar VM, Peram MR, Patil S, Bhat KG, Diwan PV (2019) Effect of Resveratrol on biofilm formation and virulence factor gene expression of Porphyromonas gingivalis in periodontal disease. APMIS 127:187-195. https://doi.org/10.1111/ apm. 12930

15. Lin YL, Chang HC, Chen TL, Chang JH, Chiu WT, Lin JW, Chen RM (2010) Resveratrol protects against oxidized LDL-induced breakage of the blood-brain barrier by lessening disruption of tight junctions and apoptotic insults to mouse cerebrovascular endothelial cells. J Nutr 140:2187-2192

16. Rege SD, Kumar S, Wilson DN, Tamura L, Geetha T, Mathews ST, Huggins KW, Broderick TL, Babu JR (2013) Resveratrol protects the brain of obese mice from oxidative damage. Oxid Med Cell Longev 2013:419092

17. Soscia SJ, Kirby JE, Washicosky KJ, Tucker SM, Ingelsson M, Hyman B, Burton MA, Goldstein LE, Duong S, Tanzi RE, Moir RD (2010) The Alzheimer's disease-associated amyloid b-protein is an antimicrobial peptide. PLoS ONE 5:e9505

18. Kim HJ, Lee KW, Lee HJ (2007) Protective effects of piceatannol against beta-amyloid-induced neuronal cell death. Annal N Y Acad Sci 1095:473-482

19. Siddiqui MA, Saquib Q, Ahamed M, Ahmad J, Al-Khedhairy AA, Abou-Tarboush FM, Musarrat J (2011) Effect of transresveratrol on rotenone-induced cytotoxicity in human breast adenocarcinoma cells. Toxicol Int 18:105-110. https://doi. org/10.4103/0971-6580.84261

20. Akyuva Y, Nazıroğlu M (2020) Resveratrol attenuates hypoxiainduced neuronal cell death, inflammation and mitochondrial oxidative stress by modulation of TRPM2 channel. Sci Rep 10:6449. https://doi.org/10.1038/s41598-020-63577-5

21. Holden JA, Attard TJ, Laughton KM, Mansell A, O'BrienSimpson NM, Reynolds EC (2014) Porphyromonas gingivalis lipopolysaccharide weakly activates M1 and M2 polarized mouse macrophages but induces inflammatory cytokines. Infect Immun 82:4190-4203. https://doi.org/10.1128/IAI.02325-14

22. Bahar B, O'Doherty JV, Smyth TJ, Sweeney T (2016) A comparison of the effects of an Ascophyllum nodosum ethanol extract and its molecular weight fractions on the inflammatory immune gene expression in-vitro and ex-vivo. Innov Food Sci Emerg Technol 37:276-285. https://doi.org/10.1016/j.ifset.2016.07.027

23. Zhang R, Miller RG, Gascon R, Champion S, Katz J, Lancero M, Narvaez A, Honrada R, Ruvalcaba D, McGrath MS (2009) Circulating endotoxin and systemic immune activation in sporadic amyotrophic lateral sclerosis (sALS). J Neuroimmunol 206:121124. https://doi.org/10.1016/j.jneuroim.2008.09.017

24. Mogensen TH (2009) Pathogen recognition and inflammatory signaling in innate immune defenses. Clin Microbiol Rev 22:240 273. https://doi.org/10.1128/CMR.00046-08

25. Kinney JS, Morelli T, Oh M, Braun TM, Ramseier CA, Sugai JV, Giannobile WV (2014) Crevicular fluid biomarkers and periodontal disease progression. J Clin Periodontol 41:113-120. https ://doi.org/10.1111/jcpe.12194

26. Cheng $\mathrm{R}, \mathrm{Wu} \mathrm{Z}$, $\mathrm{Li} \mathrm{M}$, Shao M, Hu T (2020) Interleukin- $1 \beta$ is a potential therapeutic target for periodontitis: a narrative review. Int J Oral Sci 12:2. https://doi.org/10.1038/s41368-019-0068-8

27. Harding A, Gonder U, Robinson SJ, Crean S, Singhrao SK (2017) Exploring the association between Alzheimer's disease, oral health, microbial endocrinology and nutrition. Front Aging Neurosci 9:398. https://doi.org/10.3389/fnagi.2017.00398

28. Harding A, Robinson S, Crean S, Singhrao SK (2017) Can better management of periodontal disease delay the onset and progression of Alzheimer's disease? J Alzheimers Dis 58:337-348. https ://doi.org/10.3233/JAD-170046

29. Salehi B, Mishra AP, Nigam M, Sener B, Kilic M, Sharifi-Rad M, Fokou PVT, Martins N, Sharifi-Rad J (2018) Resveratrol: a double-edged sword in health benefits. Biomedicines 6:91. https ://doi.org/10.3390/biomedicines6030091 
30. Matsuda S, Nakagawa Y, Tsuji A, Kitagishi Y, Nakanishi A, Murai $\mathrm{T}$ (2018) Implications of PI3K/AKT/PTEN signaling on superoxide dismutases expression and in the pathogenesis of Alzheimer's disease. Diseases 6:28. https://doi.org/10.3390/diseases6020028

31. Meraz-Ríos MA, Franco-Bocanegra D, Toral Rios D, CamposPeña V (2014) Early onset Alzheimer's disease and oxidative stress. Oxid Med Cell Longev 2014:375968

32. Ahmad F, Singh K, Das D, Gowaikar R, Shaw E, Ramachandran A, Rupanagudi KV, Kommaddi RP, Bennett DA, Ravindranath V (2017) Reactive oxygen species-mediated loss of synaptic Akt1 signaling leads to deficient activity-dependent protein translation early in Alzheimer's disease. Antioxid Redox Sign 27:1269-1280. https://doi.org/10.1089/ars.2016.6860

33. Tian Y, Song W, Li D, Cai L, Zhao Y (2019) Resveratrol as a natural regulator of autophagy for prevention and treatment of cancer. Onco Targets Ther 12:8601-8609. https://doi.org/10.2147/ OTT.S213043

34. Xu F, Na L, Li Y, Chen L (2020) Roles of the PI3K/AKT/mTOR signalling pathways in neurodegenerative diseases and tumours. Cell Biosci 10:54. https://doi.org/10.1186/s13578-020-00416-0

35. Antico Arciuch VG, Galli S, Franco MC, Lam PY, Cadenas E, Carreras MC, Poderoso JJ (2009) Akt1 intramitochondrial cycling is a crucial step in the redox modulation of cell cycle progression. PLoS ONE 4:e7523. https://doi.org/10.1371/journal.pone.00075 23

36. Franke TF, Yang SI, Chan TO, Datta K, Kazlauskas A, Morrison DK, Kaplan DR, Tsichlis PN (1995) The protein kinase encoded by the Akt proto-oncogene is a target of the PDGF-activated phosphatidylinositol 3-kinase. Cell 2:727-736

37. Murdock JL, Núñez G (2016) TLR4: The winding road to the discovery of the LPS receptor. J Immunol 197:2561-2562. https ://doi.org/10.4049/jimmunol.1601400

38. Androulidaki A, Iliopoulos D, Arranz A, Doxaki C, Schworer S, Zacharioudaki V, Margioris AN, Tsichlis PN, Tsatsanis C (2009) The kinase Akt1 controls macrophage response to lipopolysaccharide by regulating microRNAs. Immunity $31: 220-231$. https ://doi.org/10.1016/j.immuni.2009.06.024

39. Beurel E, Grieco SF, Jope RS (2015) Glycogen synthase kinase-3 (GSK3): regulation, actions, and diseases. Pharmacol Ther 148:114-131

40. Souder DC, Anderson RM (2019) An expanding GSK3 network: implications for aging research. Geroscience 41:369-382

41. Jope RS, Cheng Y, Lowell JA, Worthen RJ, Sitbon YH, Beurel E (2017) Stressed and inflamed, can GSK3 be blamed? Trends Biochem Sci 42:180-192. https://doi.org/10.1016/j.tibs.2016.10.009
42. Martin M, Rehani K, Jope RS, Michalek SM (2005) Toll-like receptor-mediated cytokine production is differentially regulated by glycogen synthase kinase 3. Nat Immunol 6:777-784

43. Buller CL, Loberg RD, Fan MH, Zhu Q, Park JL, Vesely E, Inoki K, Guan KL, Brosius FC (2008) A GSK-3/TSC2/mTOR pathway regulates glucose uptake and GLUT1 glucose transporter expression. Am J Physiol Cell Physiol 295:C836-C843

44. Hoshi M, Takashima A, Noguchi K, Murayama M, Sato M, Kondo S, Saitoh Y, Ishiguro K, Hoshino T, Imahori K (1996) Regulation of mitochondrial pyruvate dehydrogenase activity by tau protein kinase I/glycogen synthase kinase 3beta in brain. Proc Natl Acad Sci USA 93:2719-2723

45. Graves DT, Milovanova TN (2019) Mucosal immunity and the FOXO1 transcription factor. Front Immunol 10:2530. https://doi. org/10.3389/fimmu.2019.02530

46. Manolopoulos K, Klotz L, Korsten P, Bornstein SR, Barthel A (2010) Linking Alzheimer's disease to insulin resistance: the FoxO response to oxidative stress. Mol Psychiatry 15:1046-1052. https://doi.org/10.1038/mp.2010.17

47. Li S, Dong G, Moschidis A, Ortiz J, Benakanakere MR, Kinane DF, Graves DT (2013) P. gingivalis modulates keratinocytes through FOXO transcription factors. PLoS ONE 8:e78541. https ://doi.org/10.1371/journal.pone.0078541

48. Inglés M, Gambini J, Miguel MG, Bonet-Costa V, Abdelaziz KM, El Alami M, Viña J (2014) PTEN mediates the antioxidant effect of resveratrol at nutritionally relevant concentrations. Biomed Res Int 2014:580852. https://doi.org/10.1155/2014/580852

49. Ortega-Molina A, Efeyan A, Lopez-Guadamillas E, Muñoz-Martin M, Gómez-López G, Cañamero M, Mulero F, Pastor J, Martinez S, Romanos E, Mar Gonzalez-Barroso M, Rial E, Valverde AM, Bischoff JR, Serrano M (2012) Pten positively regulates brown adipose function, energy expenditure, and longevity. Cell Metabol 15:382-394

50. Spanier G, Xu H, Xia N et al (2009) Resveratrol reduces endothelial oxidative stress by modulating the gene expression of superoxide dismutase 1 (SOD1), glutathione peroxidase 1 (GPx1) and NADPH oxidase subunit (Nox4). J Physiol Pharmacol 60:111-116

Publisher's Note Springer Nature remains neutral with regard to jurisdictional claims in published maps and institutional affiliations. 